This document is published in:

IEEE Transactions on Terahertz Science and Technology, vol. 3, n. 4, July 2013. Avalaible on: http://dx.doi.org/10.1109/TTHZ.2013.2260374

(C) 2013 IEEE. Personal use of this material is permitted. Permission from IEEE must be obtained for all other uses, in any current or future media, including reprinting/republishing this material for advertising or promotional purposes, creating new collective works, for resale or redistribution to servers or lists, or reuse of any copyrighted component of this work in other works. 


\title{
Continuous wave sub-THz photonic generation with ultra-narrow linewidth, ultra-high resolution, full frequency range coverage and high long-term frequency stability
}

\author{
A. R. Criado, Student Member, IEEE, C. de Dios, E. Prior, G. H. Döhler, S. Preu, S. Malzer, H. Lu, A. \\ C. Gossard, and P. Acedo, Member, IEEE
}

\begin{abstract}
We report on a photonic system for generation of high quality Continuous Wave (CW) sub-THz signals. The system consists on a Gain-Switching-based Optical Frequency Comb Generator (GS-OFCG), a two-optical-modes selection mechanism and a n-i-pn-i-p superlattice photomixer. As mode selection mechanism, both selective tunable optical filtering using FabryPerot Tunable Filters (FPTF) and Optical Injection Locking (OIL) are evaluated. The performance of the reported system surpasses in orders of magnitude the performance of any commercially available optical $\mathrm{mm}$-wave and sub- $\mathrm{THz}$ generation system in a great number of parameters. It matches and even overcomes those of the best commercially available electronic THz generation systems. The performance parameters featured by our system are: linewidth $<10 \mathrm{~Hz}$ at $120 \mathrm{GHz}$, complete frequency range coverage $(60-140 \mathrm{GHz})$ with a resolution in the order of $0.1 \mathrm{~Hz}$ at $120 \mathrm{GHz}\left(10^{-12}\right.$ of generated frequency), and high long term frequency stability ( $5 \mathrm{~Hz}$ deviation over one hour). Most of these values are limited by the measurement instrumentation accuracy and resolution, thus the actual values of the system could be better than the reported ones. The frequency can be extended straightforwardly up to $1 \mathrm{THz}$ extending the OFCG frequency span. This system is compact, robust, reliable, and offers a very high performance, especially suited for sub-THz photonic Local Oscillators and high resolution spectroscopy.
\end{abstract}

Index Terms-Optical Frequency Comb, THz Local Oscillator, narrow linewidth $\mathrm{THz}$ generation, $\mathrm{THz}$ photonic generation, Optical Injection Locking

Manuscript received December 12, 2012. Work supported by the Spanish Ministry of Science and Technology through the project TEC2009-14525C02-02. The work by Á.R. Criado has been supported by the Spanish Ministry of Science and Technology under the FPI Program, Grant\# BES2010-030290.

Á. R. Criado, C. de Dios, E. Prior and P. Acedo are with the Electronics Technology Department, Universidad Carlos III de Madrid, Leganés, Madrid 28911 Spain (e-mail: acriado@ing.uc3m.es).

G. H. Döhler is with the Max Planck Institute for the Science of Light, Guenther-Scharowsky-Str. 1/ Bldg. 24, 91058 Erlangen, Germany.

S. Preu and S. Malzer are with the Lehrstuhl für Angewandte Physik, Friedrich-Alexander-Universität Erlangen-Nürnberg, 91058 Erlangen, Germany.

H. Lu and A.C. Gossard are with the Materials Dept. University of California, Santa Barbara, Santa Barbara, CA USA

\section{INTRODUCTION}

$\mathrm{T}$ HE study of the sub- $\mathrm{THz}$ and $\mathrm{THz}$ frequency ranges has experienced a great development in the last years [1]. There is a great number of emerging applications in this frequency range, namely, imaging (for security [2] and biomedical [3] purposes among others), short-range high datarate communications [4] and spectroscopy [5], [6]. Nevertheless, spectroscopy applications have been the ones that have driven the $\mathrm{THz}$ development since the 70's due to their importance in radioastronomy [7]. Even today, it remains the main field of application within the $\mathrm{THz}$ range, widely used in security [2], material identification and inspection [8], biomedicine [9] and radioastronomy [10], [11].

Nowadays, the $\mathrm{THz}$ research focus is mainly oriented to generation and detection systems that would allow full exploitation of all these applications. This requires the development of technologies providing compactness, reasonable cost, stability, robustness and room temperature operation. Specifically, if the application is related to Continuous Wave (CW) spectroscopy, generation systems must feature narrow linewidth and continuous tunability with a tuning resolution below the linewidth of the species under test. Moreover, a robust and reliable system with high long-term stability and frequency accuracy is desirable to properly accomplish frequency sweep operation and provide customer satisfaction.

The existing $\mathrm{THz}$ generation technologies can be classified in three groups: electronic upconversion, $\mathrm{THz}$ oscillators and optical downconversion. The electronic upconversion aims to push the frequency limits from the mm-wave region up to the $\mathrm{THz}$ region, while the optical downconversion relies mainly on Difference Frequency Generation (DFG) to generate signals down to the $\mathrm{THz}$ range [7]. The $\mathrm{THz}$ oscillators make use of several technologies and are able to directly generate $\mathrm{THz}$ radiation.

Among the compact sources able to generate coherent $\mathrm{THz}$ radiation with the different approaches mentioned above, the following technologies should be highlighted: electronic upconversion using Schottky multipliers [12]; direct $\mathrm{THz}$ 
generation with Resonant Tunneling Diodes (RTD) [13] or $\mathrm{THz}$ Quantum Cascade Lasers (THz-QCL) [14], and optical downconversion based on DFG using photomixers.

Schottky multipliers are a commercial solution capable of providing high performance $\mathrm{THz}$ signals up to $2.7 \mathrm{THz}$ with a narrow linewidth (Hz range), comparatively good power levels (around $100 \mu \mathrm{W}$ at $1 \mathrm{THz}$ ), and continuous tunability within a limited frequency range determined by the employed waveguides [12]. However, the generation relies on a radiofrequency $(\mathrm{RF})$ reference that is multiplied; this worsens the linewidth and phase noise performance as the multiplication order increases [15].

RTDs operating up to $1.1 \mathrm{THz}$ have been demonstrated [13] and they are a promising technology with high integration potential. However, the generated $\mathrm{CW}$ signal shows a wider linewidth (in the order of $10 \mathrm{MHz}$ [16]) and the tunability is very limited (about $4 \%$ of the central frequency [17]). THzQCLs provide a very high signal power (up to $140 \mathrm{~mW}$ in CW operation [18]), but they hardly operate below $2 \mathrm{THz}$ (the minimum demonstrated frequency is $0.8 \mathrm{THz}$, achieved by applying external magnetic fields [7]). They also need cryogenic cooling to offer the mentioned high power in the $\mathrm{THz}$ range [19]. Moreover, they can only provide narrow linewidths (in the range of 100's Hz) by making use of complex Phase Locked Loop systems [20], and have a very limited tunability, with a record value of $8.6 \%(330 \mathrm{GHz})$ around the center frequency by mechanical means [21].

$\mathrm{THz}$ generation from DFG in photomixers, although it offers a slightly lower power than other solutions (up to 24 $\square \mathrm{W}$ at $0.914 \mathrm{THz}$ ) [22], is able to offer continuous tunability over a large frequency range (up to $1.53 \mathrm{THz}$ for photodiodebased photomixers) in compact systems [23]. Additionally, they offer the possibility of distributing the optical signal using optical fiber with very low losses $(0.2 \mathrm{~dB} / \mathrm{km})$ and Electromagnetic Interference (EMI) immunity to one or several remote photomixers where the $\mathrm{THz}$ generation is accomplished. This means that the mixing process can be carried out remotely. This is a really interesting feature for remote spectroscopy, remote sensing and Local Oscillator (LO) distribution in radioastronomy heterodyne receivers [11]. With respect to the integration potential, a great effort is placed today and Photonic Integrated Circuits (PICs) for sub$\mathrm{THz}$ generation have been already implemented [24].

In this scenario, the electronic upconversion using Schottky diodes and the optical downconversion using DFG with photomixers are the most suitable approaches for a compact and good performance source below $2 \mathrm{THz}$. There exist commercial solutions for both technologies [12], [25] but nowadays Schottky diodes are preferred because of their better characteristics in terms of output power, linewidth, frequency resolution, long term stability and frequency accuracy.

In this work we are going to discuss a DFG based system for narrow linewidth, large frequency coverage for sub-THz and $\mathrm{THz}$ generation. These optical architectures are based on photomixing of two optical modes to produce a signal with a frequency equal to the frequency spacing between those modes. Hence, a simplified DFG scheme consists of an optical source providing the two required optical frequencies and a photomixer. There are several ways to obtain the two optical modes. Two single-mode lasers can be used, offering continuous tunability. However, in order to obtain a narrowlinewidth $\mathrm{THz}$ signal, the lasers have to be very well stabilized in frequency and they have to be locked between each other by complex Optical Phase Locked Loops (OPLL) to attain coherence between the optical lines. Even in those cases, the linewidth and tunability resolution of the synthesized $\mathrm{THz}$ signal is typically in the $\mathrm{MHz}$ range [25]. If a two-mode laser is used, it can offer much better mode coherence, hence a better linewidth performance without OPLLs, but there is no tunability or it is very limited [26]. More interesting are the multimode sources (i.e. Optical Frequency Comb Generators (OFCG)), such as Mode-Locked Laser Diodes (MLLD) [27] or tunable OFCGs. An OFCG presents high coherence between longitudinal modes, and the linewidth and phase noise characteristics of the synthesized signals can be very good, but an additional stage for the selection of the two modes is required. MLLDs can be integrated as PICs but the frequency tunability of the generated signal is discrete, in frequency steps corresponding to the spacing between modes [28]. Tunable benchtop OFCGs are able to provide tunability of the frequency spacing between modes and then, continuous tunability over the whole range, overcoming the issue presented by MLLDs. Between the tunable OFCGs, some schemes based on Gain Switching (GS) [29], [30] have been recently reported, featuring a more compact and cost-effective setup than other benchtop OFCGs, as fewer components are employed to produce an equivalent optical span, all of them reliable and commercially available.

When a multimode OFCG source is considered, a mode selection stage is often included in the system to provide coarse frequency tunability. For this block of the system, two basic approaches can be followed. The first one is selective optical filtering, using Arrayed Waveguide Gratings (AWG) [31] or high-selective Fabry-Perot tunable filters (FPTF) [27], [28], [30], [32]. An alternative mode selection mechanism is the use of Optical Injection Locking (OIL) techniques [33].

Every DFG system is completed with the photomixing device responsible for the photonic-to-RF frequency conversion. Devices based on $\mathrm{p}-\mathrm{i}-\mathrm{n}$ structures are preferred to photoconductors due to their higher available output power, although they offer a lower bandwidth, around $1.53 \mathrm{THz}$ [23]. Examples of these $\mathrm{p}-\mathrm{i}-\mathrm{n}$ devices are Uni-Travelling-Carrier photodiodes (UTC-PD) [31], Travelling-Wave UniTravelling-Carrier (TW-UTC-PD) [23] and n-i-pn-i-p superlattice photomixers [30], [34], [35]. The latter take advantage of a superlattice of $\mathrm{p}-\mathrm{i}-\mathrm{n}$ diodes allowing for independent optimization of both transit and RC times, thus overcoming the trade-off usually present in most highbandwidth photodiodes.

In this paper, we present a system that offers the advantages 
of the optical downconversion (i.e. complete and continuous frequency range coverage from 60 to $140 \mathrm{GHz}$ with potential extension to several hundreds of $\mathrm{GHz}$ and optical fiber distribution) and is able to match and even surpass the linewidth, tunability resolution and frequency stability of the best available electronic $\mathrm{THz}$ sources (i.e. Schottky multiplier chains). This is achieved with a compact and simple scheme that is also able to provide a robust and reliable system.

The system is a DFG scheme consisting of a GS based OFCG, a selective filtering stage and a n-i-pn-i-p superlattice photomixer. Both optical filtering using FPTF [30] and OIL are evaluated and compared to determine the most suitable optical filtering method. The sub- $\mathrm{THz}$ output signal is evaluated in terms of linewidth, power, tunability resolution and range, and power and frequency stability. The resulting system and its performance make it a potential candidate for high resolution $\mathrm{CW}$ spectroscopy able to compete with the best electronic $\mathrm{THz}$ commercially available systems.

\section{OPTICAL MULTIMODE SOURCE: GAIN-SWITCHING-BASED OPTICAL FREQUENCY COMB GENERATOR}

\section{A. Principle of operation}

One of the more compact and efficient schemes for the generation of multimode sources (Optical Frequency Combs) relies on the use of a laser under GS modulation as seed [29]. As it can be seen in Fig. 1, a basic version of a Gain Switching based OFCG (GS-OFCG) consists of a Discrete Mode (DM) laser that is modulated under GS regime and then phase modulated with a driving signal synchronized in phase with the GS modulating signal [30].

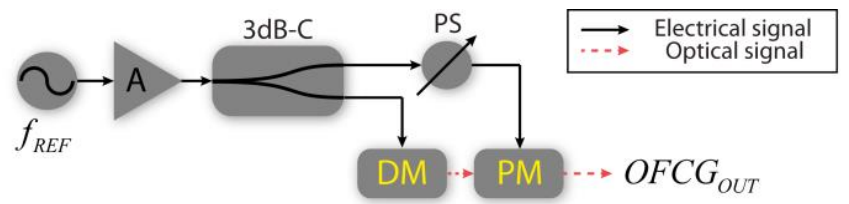

Fig. 1. Scheme of the GS-based OFCG. 3dB-C ( $3 \mathrm{~dB}$ electrical splitter), DM (Discrete Mode laser), PS (Electrical Phase Shifter), PM (Optical Phase Modulator)

\section{B. Performance}

In order to characterize the phase noise degradation of the RF reference signal $\left(f_{\text {REF }}\right)$ after passing through the GS-OFCG and its non-linear mechanisms, in Fig. 2 is shown the Single Side-Band (SSB) noise of both the reference signal (directly measured at the output of the RF amplifier, $A$, in Fig. 1) and the photodetected output of the GS-OFCG $\left(O F C G_{\text {OUT }}\right.$ in Fig. 1). The parameters of the GS modulation are $\mathrm{I}_{\mathrm{DM}}=60 \mathrm{~mA}, \mathrm{f}_{\mathrm{REF}}$ $=10 \mathrm{GHz}$ and $\mathrm{P}_{\mathrm{REF}}=27 \mathrm{dBm}$. The measurement floor is shown (Displayed Average Noise Level, DANL). Both signals are measured with the same dynamic range and amplification chain. There is no significant degradation at low and high frequency offsets (below $100 \mathrm{kHz}$ and above $10 \mathrm{MHz}$ ). However, two differences are observed. Firstly, a spurious signal at about $9 \mathrm{kHz}$ that is present in the reference does not appear in the GS-OFCG output. Secondly, a $250 \mathrm{kHz}$ spurious signal is observed in the GS-OFCG output. Around this last spurious signal, a certain degradation of the actual signal can be observed. One possible explanation for this is the high power applied to the optical phase modulator. Nevertheless, it is not significant at all as can be concluded by the Root-MeanSquare (RMS) integrated jitter values over the $20 \mathrm{kHz}-80$ $\mathrm{MHz}$ frequency region. The values are $70.97 \mathrm{fs}$ for the reference signal and 76.05 fs for the OFCG-GS output. The DANL has a jitter of 25 fs. The Full Width at Half Maximum (FWHM) of both signals have the same value of about $4 \mathrm{~Hz}$ (measured with a RBW $=1 \mathrm{~Hz}$ ) (Fig. 2, inset), which basically corresponds to the Local Oscillator of the Electrical Spectrum Analyzer (ESA) rather than to the employed reference signal.

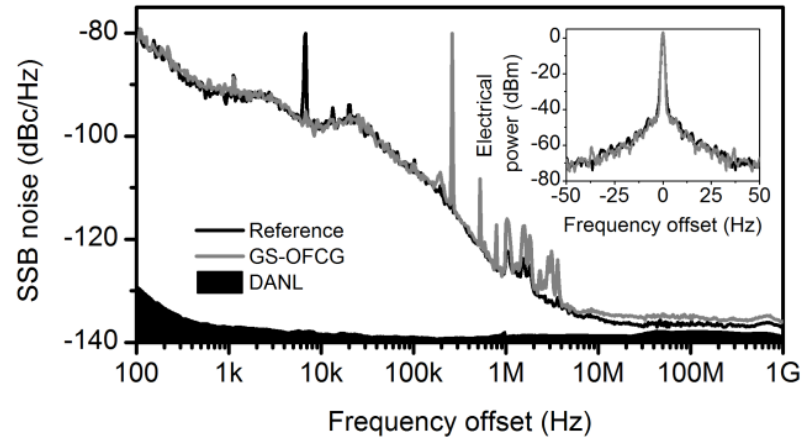

Fig. 2. SSB noise measurements for Fig.1. Inset: measured electrical spectra for both signals. Black trace: reference signal at $10 \mathrm{GHz}$; grey trace: photodetected OFCG output at $10 \mathrm{GHz}$. Four samples average for each trace (see text for details).

For the characterization of the GS-OFCG we investigated the evolution of the optical spectra with respect to changes in the modulation power $\left(\mathrm{P}_{\mathrm{REF}}\right)$, in the phase difference between both GS and phase modulating signals $\left(\phi_{\mathrm{MOD}}\right)$ and in the bias current of the DM laser ( $\mathrm{I}_{\mathrm{DM}}$, see Fig.1).

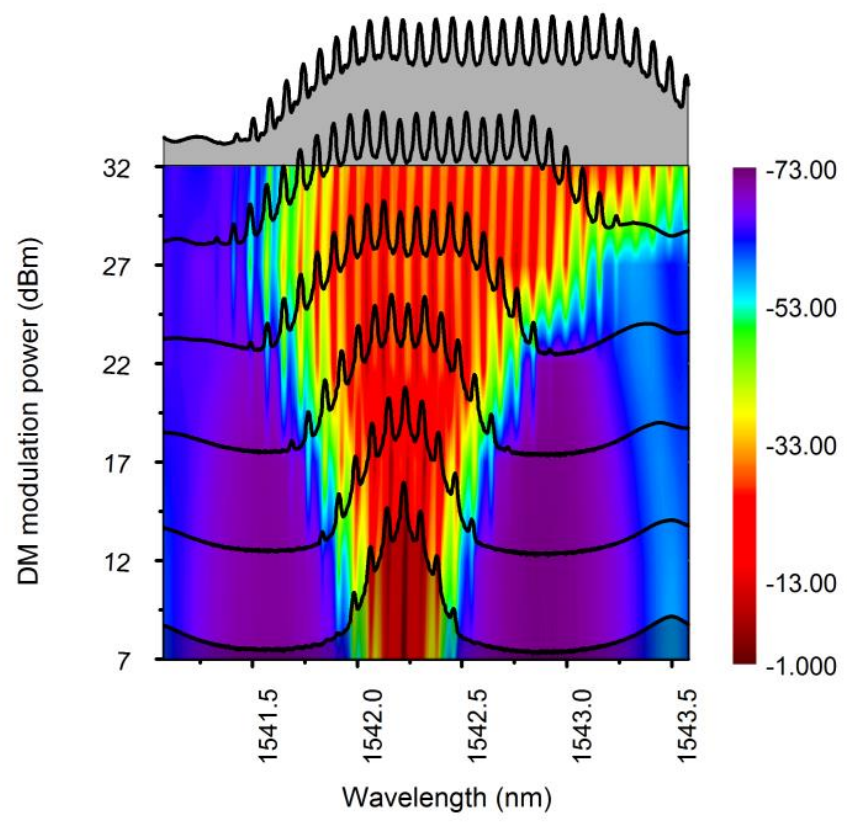

Fig. 3. OFCG optical spectra ( $\mathrm{Z}$ axis: optical power $(\mathrm{dBm})$ ). Evolution with the modulation power of the DM laser 
The optical spectra of the GS-OFCG, as a function of $\mathrm{P}_{\mathrm{REF}}$, are shown in Fig. 3. As expected, higher values of $\mathrm{P}_{\mathrm{REF}}$ provide a wider optical comb, and a flat comb with up to 23 lines (10 $\mathrm{GHz}$ apart) within a $10 \mathrm{~dB}$ bandwidth can be generated. However, the maximum value of $\mathrm{P}_{\mathrm{REF}}$ is limited by the maximum modulating power the DM and the PM can admit. In our case, this value corresponds to approximately $32 \mathrm{dBm}$.

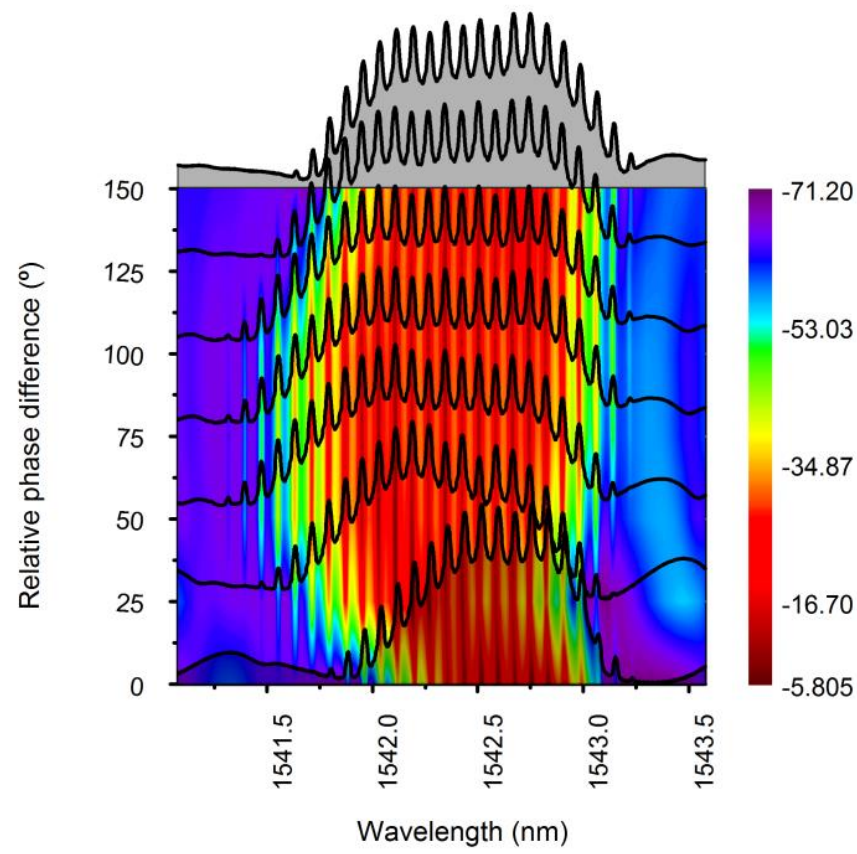

Fig. 4. OFCG optical spectra ( $\mathrm{Z}$ axis: optical power $(\mathrm{dBm})$ ). Evolution with the relative phase difference between the DM modulation signal and the optical phase modulator driving signal.

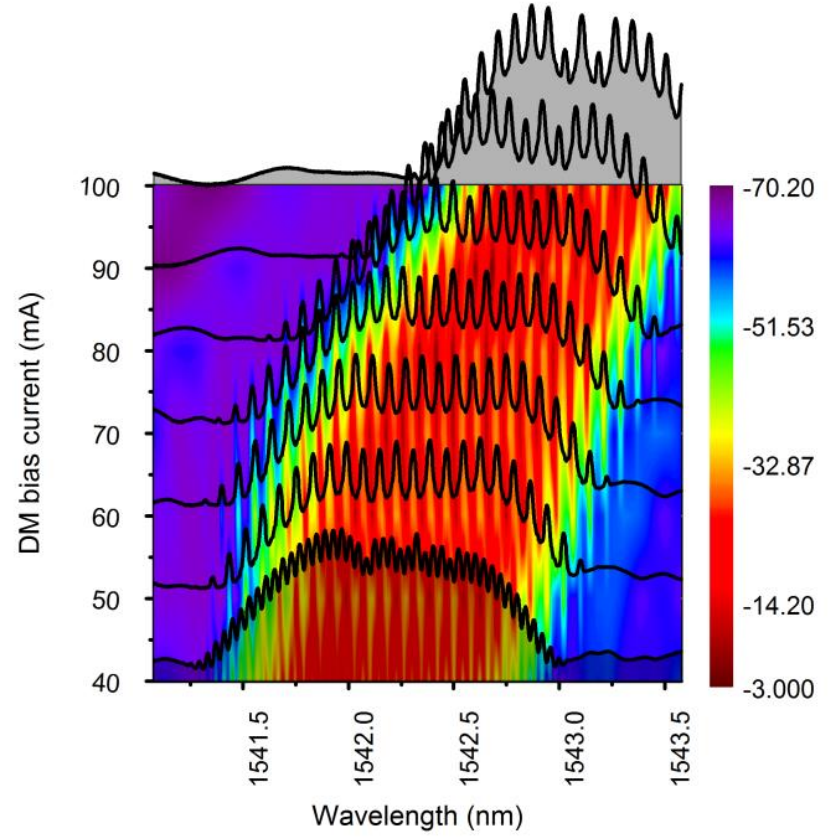

Fig. 5. OFCG optical spectra ( $\mathrm{Z}$ axis: optical power $(\mathrm{dBm})$ ). Evolution with the bias modulation of the DM laser.

The evolution of the GS-OFCG with the change in $\phi_{\mathrm{MOD}}$ is represented in Fig. 4, which demonstrates the importance of this parameter in the optical span and flatness adjustments of the GS-OFCG output. This is the reason why an electrical phase trimmer or phase shifter (PS) is required in the scheme of the GS-OFCG (see Fig.1).

Finally, Fig. 5 shows how the optical spectra change with $\mathrm{I}_{\mathrm{DM}}$. In this case, it is clearly shown how the spectra evolve from GS modulation (lower $\mathrm{I}_{\mathrm{DM}}$ ) to linear modulation (higher $I_{D M}$ ), when the effect of the PM is dominant and the phase modulation side bands appear. It is important to note that the value $\mathrm{I}_{\mathrm{DM}}$ has to be carefully selected to provide proper GS regime and maximize the optical span [36].

\section{GS-OFCG Continuous Tunability}

The tunability of the GS-OFCG (frequency spacing between modes) is basically limited by the bandwidth of its components. The DM laser employed in this work has a usable bandwidth of around $20 \mathrm{GHz}$, thus being able to theoretically provide GS operation up to that frequency. In our case, the limiting factor is the PM, with a bandwidth around $10 \mathrm{GHz}$ (the $P S$ and power amplifier $A$ (Fig. 1) have a bandwidth of 18 $\mathrm{GHz}$ ).

For our GS-OFCG scheme, the frequency spacing between modes $\left(\mathrm{f}_{\mathrm{REF}}\right)$ can be tuned directly using the reference $\mathrm{CW}$ generator. Nevertheless, due to the dependence on $\phi_{\mathrm{MOD}}$, significant frequency detuning values (above $10 \mathrm{MHz}$ ), require a re-tuning of $\phi_{\mathrm{MOD}}$. In this work, manual re-tuning has been used, although a control loop using a voltage-controlled electrical phase shifter would provide automatic control of this parameter.

\section{Optical Comb Frequency Span}

The basic GS-OFCG presented in this paper includes up to 23 modes within a $10 \mathrm{~dB}$-bandwidth for a $220 \mathrm{GHz}$ frequency span. An increase in the number of modes can be accomplished by increasing the modulation depth of the signal inducing the GS regime in the DM laser and/or the phase modulator RF input (see Fig. 3). However, these devices have a maximum input power that determines the maximum optical span that a basic GS-OFCG, like that on Fig. 1, is able to provide.

Nevertheless, there exist many techniques to broaden the optical span of an OFCG that can be added to the basic GSOFCG scheme of Fig. 1 in order to provide a GS-OFCG with an optical span above $1 \mathrm{THz}$. Recent works on the expansion of OFCG consider the use of cascaded phase modulators and intensity modulator elements [37], resulting in a flat optical spectra exceeding $3 \mathrm{THz}$ [38]. Other techniques are based on polarization modulation [39]. Another approach is the exploitation of the nonlinear propagation of light travelling along special highly nonlinear optical fibers [40]. Interferometric nonlinear propagation of light has also been studied as shapers for pulsed optical sources. Results on the capability of Sagnac interferometers to expand the optical spectra have also been reported [41], showing a good potential as a new OFCG expander technique. Hence, it is clear that the broadening of the GS-OFCG detailed in this work can be 
accomplished and this would expand the frequency synthesis of the presented system to the $\mathrm{THz}$ domain straightforwardly.

\section{SELECTION OF TWO OPTICAL MODES: HIGH-SELECTIVITY FABRY-PEROT TUNABLE OPTICAL FIBER FILTERS}

\section{A. Principle of operation}

One of the most widely used methods to select the two required optical modes from a multimode source in DFG schemes is to use selective filtering. This can be accomplished with Arrayed Waveguided Gratings (AWGs) [31], or highselective Fabry-Perot Tunable Filters (FPTF) [27], [28], [30], [32]. In this section we are going to evaluate the use of the second type of components (FPTF) for mode selection. It is important to note that in the case of selective filtering optical gain is usually needed to compensate the insertion losses of the filters [27]. Fig. 6 shows the experimental scheme for the generation of $\mathrm{THz}$ signals using FPTF.

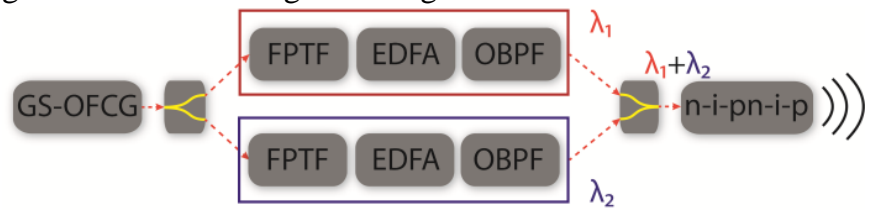

Fig. 6. THz photonic generation scheme using selective optical filtering. OFCG: Gain-Switched Comb Generator; FP-TF: Fabry-Perot Tunable Filter; EDFA: Erbium Doped Fiber Amplifier; OBPF: (optional) Optical Band-Pass Filter.

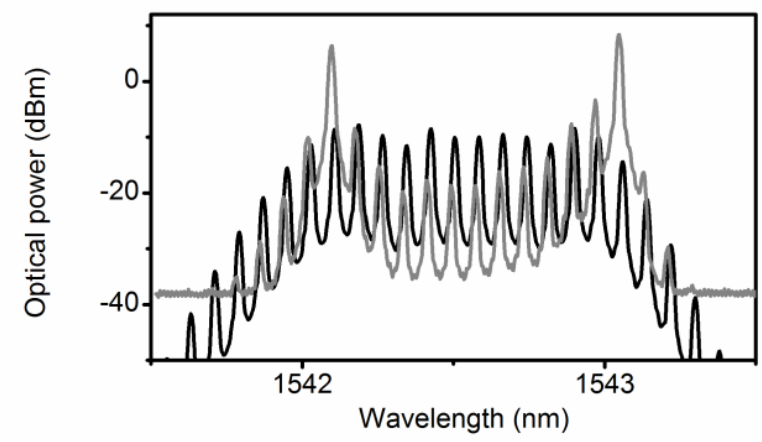

Fig. 7. Measured optical spectrum at the photomixer input using selective optical filtering (grey trace, see Fig. 6). OFCG spectrum (black trace).

A typical optical spectrum, after FPTF filtering and prior to photomixing (see Fig. 6) is shown in Fig. 7. The optical bandwidth (FWHM) of the used FPTF is about $6 \mathrm{GHz}$ at 1550 $\mathrm{nm}$, thus having a Side Mode Suppression Ratio (SMSR) of about $13 \mathrm{~dB}$ for a frequency spacing of $10 \mathrm{GHz}$. It can be also seen from Fig. 7 that there is an increase in the noise floor due to the Amplified Spontaneous Emission (ASE) of the Erbium Doped Fiber Amplifier (EDFA) required to compensate for filters insertion losses.

\section{B. Long Term Stability}

Given the high selectivity of the employed FPTF $(\mathrm{Q}=$ 3000 ), a bias voltage value of around $8 \mathrm{mV}$ applied to the piezoelectric transducer (PZT) of the FPTF, detunes its center frequency by $10 \mathrm{GHz}$. This requires highly stable voltage sources, and is one of the drawbacks of using FPTFs.
The driving voltage of the FPTF is provided in this case by a commercial voltage source (Agilent E3631A) with a resolution of $1 \mathrm{mV}$. Alternatively, a custom made dual channel DC voltage source has been designed and implemented, featuring a resolution of $20 \mu \mathrm{V}$ and theoretical output stability in the range of a few $\mathrm{ppm} /{ }^{\circ} \mathrm{C}[42]$.

The long term stability ( $60 \mathrm{~min}$ ) of the FPTFs is tested using both voltage sources. Fig. 8 shows both the wavelength drift and the room temperature drift. The wavelength drift has been measured at the output of the FPTF, using a white light source as input. From Fig. 8, it can be seen that there is a significant correlation between the room temperature drift and the voltage drift (i.e. wavelength drift). The best results are obtained with the custom made DC source, with a wavelength drift of around $0.2 \mathrm{~nm}(25 \mathrm{GHz})$ in one hour. The results conclude that a short term operation is possible with free-running FPTF, but high long term stability would require very accurate temperature control or preferably control loops for the FPTFs. The latter solution requires a very precise electronic design due to voltage values below $1 \mathrm{mV}$. Control loops for this Q-factor range have been demonstrated [43].

Finally, it must be noted that the drift of the center frequency of the FPTFs is translated to the THz synthesized signal as power drifts. However, the filters' drift have no impact in the linewidth or phase noise of the THz signal [27].

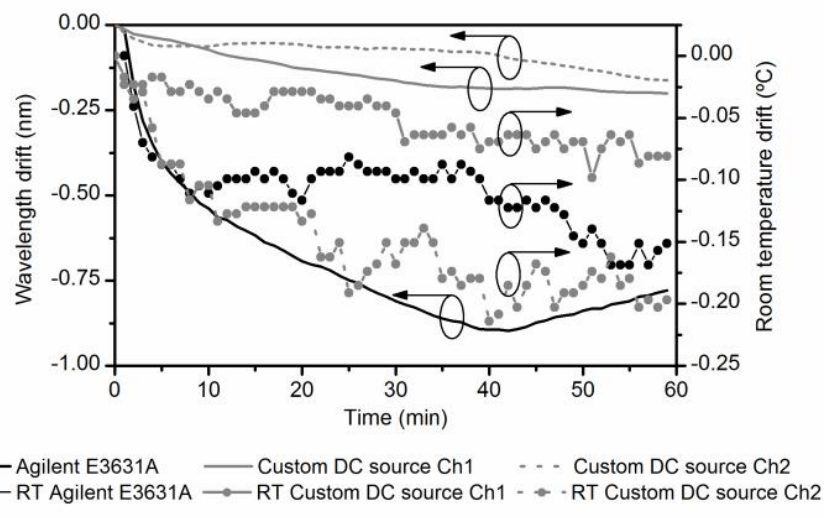

Fig. 8. Long term stability of the high selective FPTF. Wavelength drift (line traces) and room temperature drift (line + symbol traces). Black traces (commercial voltage source Agilent E3631A), grey traces (custom DC source, channels 1 and 2).

\section{SELECTION OF TWO OPTICAL MODES: OPTICAL INJECTION LOCKING}

\section{A. Principle of operation}

Another mechanism of optical mode selection from a multimode source is Optical Injection Locking (OIL) [33]. The desired optical mode of the source is injected to a slave laser, which acts both as filter and amplifier, in the sense that it is able to filter the injected mode and to provide with optical gain at the same time. As the optical gain is only applied to the selected optical mode, better values of noise floor and SMSR are achieved. When the process is performed over the two required optical modes, a very useful scheme for DFG $\mathrm{THz}$ generation can be obtained (Fig. 9). 


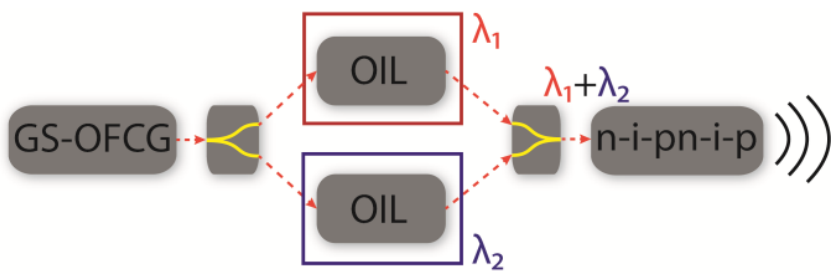

Fig. 9. THz photonic generation scheme using OIL. OFCG: Gain-Switched Comb Generator; OIL: Optical Injection Locking.

A typical optical spectrum at the input of the photomixer (Fig.11) is shown in Fig. 10. When comparing with Fig. 7, it can be clearly seen that a higher SMSR (around $30 \mathrm{~dB}$ ) and much lower values of the noise floor are obtained for same output power. This SMSR value has a negligible effect in the phase noise of the sub-THz generated signal[44].

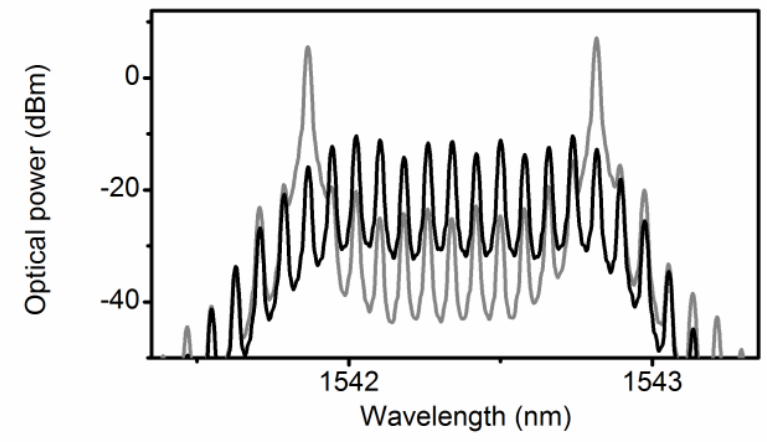

Fig. 10. Measured optical spectrum of photomixer input using OIL (grey trace) and OFCG spectrum (black trace).

\section{B. Locking behavior}

In the mode selection using optical filtering, the operation consists on tuning the center frequency of the two filters on the selected optical modes (e.g. using a voltage source to control the filters). At a first glance, the OIL seems to be a much more complex scheme. Nevertheless, we will show that the operation requirements are not very complex compared to optical filtering and performance is much better.

When the OFCG is injected into the slave laser (in this case a DFB laser), both current and especially temperature control of the DFB allow centering the slave on the selected mode from the OFCG. If the optical signal after OIL is photodetected in an ESA, the beat note between the adjacent modes of the OFCG and the slave appears. Usually, the baseband is monitored. In our case, due to the availability of adjacent modes from the OFCG, it is more convenient to monitor the region around $f_{\text {REF }}$. Fig. 11 shows how the beat note around $10 \mathrm{GHz}$ evolves as the slave laser wavelength is centered on the selected optical mode from the master laser (GS-OFCG). Proper OIL can be identified when the noise floor drastically reduces and the peak frequency $\left(f_{R E F}\right)$ increases, as it can be seen in Fig. 11.

Nevertheless, OIL is only possible for a certain amount of injected power. In this work, we will use the injection maps to evaluate the Injected Ratio per optical Mode (IRm) that is the ratio of the slave power to the power of the selected optical mode from the OFCG. The measured locking region map as a function of the two control parameters (frequency detuning and IRm) is shown in Fig. 12, where the expected V-shaped map for OIL can be observed. It must be noted that, although a wider tuning range of OIL is obtained with lower IRm, a high amount of injected power eventually produces unstable operation. Then, IRm values around 25-35 dB are preferred for our application. The estimated frequency to temperature tuning ratio for the employed DFBs is around $27.2 \mathrm{MHz} / \Omega$, referred to the standard $10 \mathrm{k} \Omega$ thermistor included in the butterfly packages of both DM and DFB lasers.

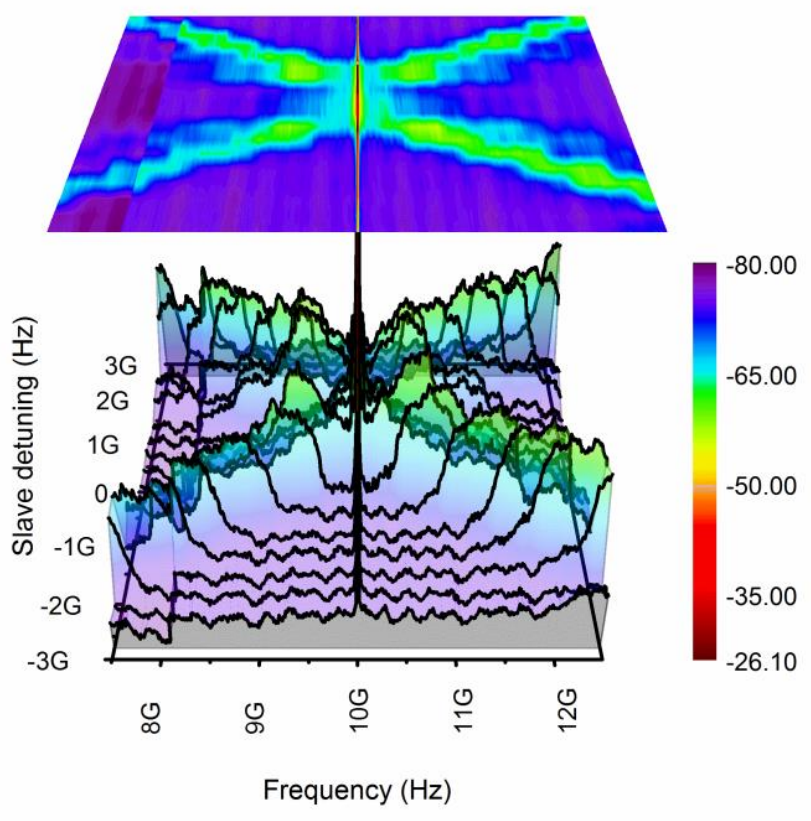

Fig. 11. OIL beat note around $10 \mathrm{GHz}(\mathrm{Span}=5 \mathrm{GHz})$. ( $\mathrm{Z}$ axis: electrical power $(\mathrm{dBm})$ ). Evolution of the electrical spectra with the frequency detuning of the slave laser. The IRm used is $35 \mathrm{~dB}$.

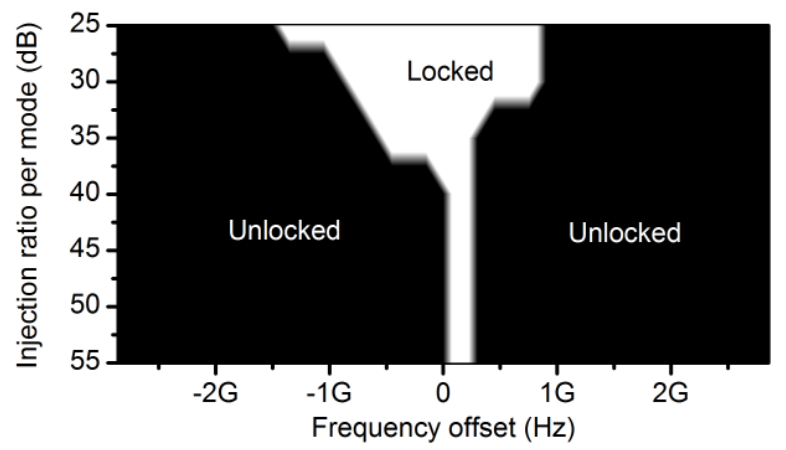

Fig. 12. Locking region map. Locking state (locked/unlocked) as a function of the frequency detuning between master and slave lasers and as a function of the IRm parameter.

\section{Long Term Stability}

In order to characterize the long term stability of the mode selection mechanism based on OIL, the beat note of the output of the slave laser (see Fig. 11) is monitored with respect to the time in the locked state. In Fig. 13 we monitored the OIL over time. It remained locked over the measured period of $1 \mathrm{hr}$. Much higher locking times are experimentally expected, as the stability depends on the temperature and current control of the 
lasers and the locking range $(2 \mathrm{GHz}$ at $\mathrm{IRm}=25 \mathrm{~dB})$. Regular laboratory-grade laser diode controllers have stability specifications of $40 \mathrm{ppm}$ in current and $0.01^{\circ} \mathrm{C}$ in temperature control over 24 hours [45]. This can be translated for our setup into a 24-hour stability of $0.005 \mathrm{~mA}$ and $5 \Omega$ (around 150 $\mathrm{MHz}$ ), amply within the OIL locking range. So, the system should remain stable for days as long as normal conditions in the setup are kept.

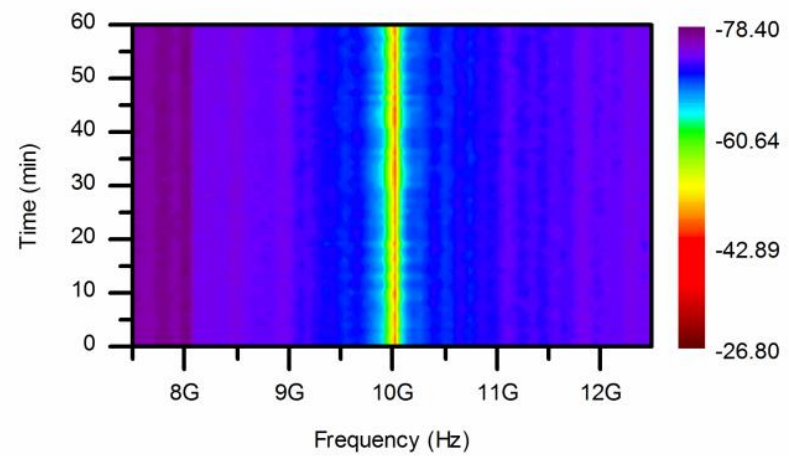

Fig. 13. OIL beat note under locked state. ( $\mathrm{Z}$ axis: electrical power $(\mathrm{dBm})$ ). Evolution of the electrical spectra with time (see text for details).

\section{PHOTOMIXER: N-I-PN-I-P SUPERLATTICE PHOTOMIXER}

The photomixer used in this $\mathrm{THz}$ photonic synthesis system is a n-i-pn-i-p superlattice photomixer [34]. A packaged version has been used, with a FC/APC optical input and a BNC bias input. The fiber-coupled photomixer consists of a three period n-i-pn-i-p superlattice photodiode that is coupled to a logarithmic-periodic broadband antenna. It covers a frequency range from below $100 \mathrm{GHz}$ to about $1.4 \mathrm{THz}$ (c.f. Fig. 14 and ref [22]]). The chip (i.e. the photomixer antenna) is attached to a hyperhemispherical lens to provide a free space THz output.

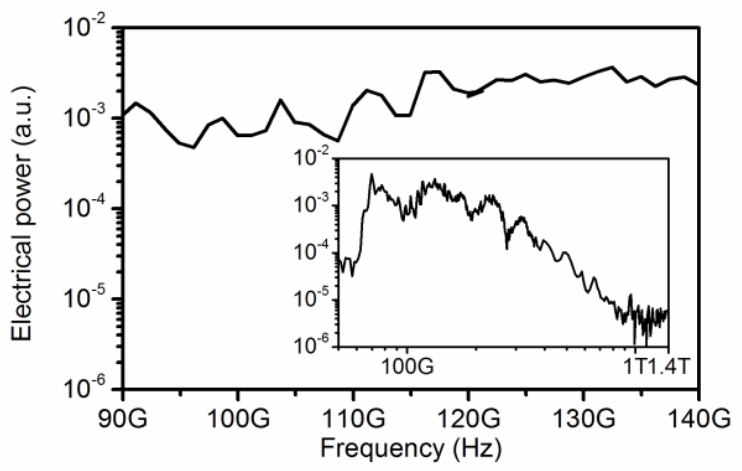

Fig. 14. Frequency response of the n-i-pn-i-p superlattice photomixer (packaged version). Inset: whole frequency response of the photomixer (Xaxis is Frequency $(\mathrm{Hz})$ and Y-Axis is Electrical power (a.u.). The generated photocurrent is $0.5 \mathrm{~mA}$.

The frequency response of the n-i-pn-i-p photomixer in the operation frequency range is depicted in Fig. 14, which also shows the whole operation bandwidth of the device in excess of $1 \mathrm{THz}$ (Fig. 14, inset).

With the optical power delivered in this work to the photomixer (around $15 \mathrm{dBm}$ ), the generated photocurrent is
$-235.5 \mu \mathrm{A}$, at a bias voltage of $-1.6 \mathrm{~V}$. Safe long-term operation of this device is expected for a photocurrent up to 2-3 $\mathrm{mA}$ where the thermal load within the device is still low. Thus much higher optical power (and up to 100 times higher $\mathrm{THz}$ output power) could be used for this work, but as the objectives were not associated to high power generation, we kept the working point far below the maximum power.

\section{CW SUB-THZ GENERATION: EXPERIMENTAL RESULTS}

\section{A. Experimental setup}

From the characterization of the two mode selection mechanisms of sections III and IV, it can be concluded that OIL provides higher performance since it offers better mode selectivity, higher optical power associated to the selected modes, less noisy optical spectra and a much better long term stability. The CW sub-THz generated signals and their characterization are presented in this section, where OIL has been used as mode selection mechanism. The experimental setup is depicted in Fig. 9. Due to the limitations in our available electrical characterization measurement system (ESA), the results are limited up to frequencies of $140 \mathrm{GHz}$.

\section{B. Ultra-narrow linewidth performance}

The CW sub-THz signal generated at $120 \mathrm{GHz}$ is shown in Fig. 15. The span and resolution bandwidth (RBW) are the minimum allowed by the ESA at this frequency range $(2.1 \mathrm{kHz}$ and $10 \mathrm{~Hz}$, respectively). The measured FWHM of the generated mm-wave signal is $10 \mathrm{~Hz}$ (Fig. 15), which corresponds to the employed RBW, thus we can conclude that the actual FWHM is $\leq 10 \mathrm{~Hz}$.

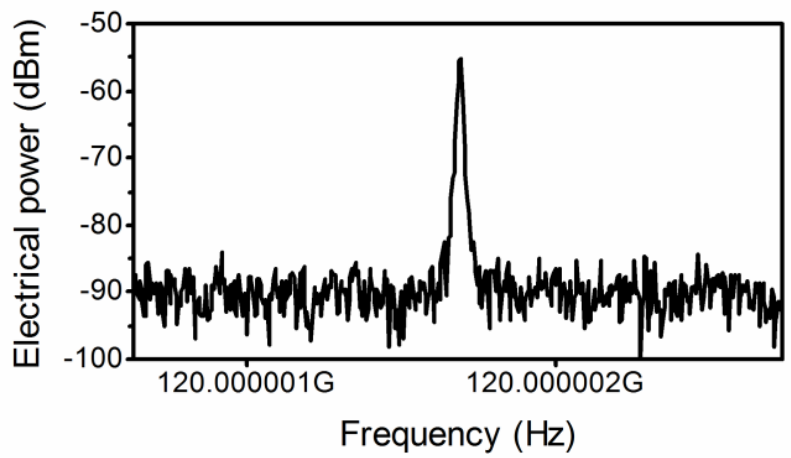

Fig. 15. Sub-THz generated signal at $120 \mathrm{GHz}$. Span $=2.1 \mathrm{kHz}, \mathrm{RBW}=10$ $\mathrm{Hz}$, sweep time $=63 \mathrm{~s}$.

This result shows that the OIL mechanism does not degrade the phase noise or FWHM of the sub-THz generated signal, providing in this sense a mode selection scheme as good as just optical filtering, where no degradation in the $\mathrm{THz}$ signal is produced [30], [32].

It is worth saying that although there is no evident degradation of the phase noise spectrum (Fig. 15) due to optical fiber instabilities of the experimental setup (Fig. 6 and 9) as would be expected from other works [46], this is because in our case (i.e. heterodyne) the phase difference between both branches (i.e. interferometric term) does not translate to the 
frequency spectrum[47]. Nevertheless, the temporal signal will still have these phase fluctuations and additional phase stabilization mechanisms must be considered for phasesensitive applications.

\section{Ultra-high resolution tunability with complete frequency range coverage}

The $\mathrm{THz}$ frequency delivered by the system at the output of the photomixer corresponds to $f_{\mathrm{THZ}}=\mathrm{n} \cdot \mathrm{f}_{\mathrm{REF}}$ where $\mathrm{n}$ is the number of modes between the two selected optical modes. The complete and quasi-continuous frequency coverage of the 60$140 \mathrm{GHz}$ band of our $\mathrm{THz}$ photonic generation system is achieved in two complementary ways. The coarse tunability (in $\mathrm{f}_{\mathrm{REF}}$ steps) is given by the selection of the different optical modes from the GS-OFCG using one of the two previously analyzed mode selection mechanisms. The fine tuning is accomplished by detuning $\mathrm{f}_{\mathrm{REF}}$. In this case, the detuning of the $\mathrm{THz}$ signal corresponds to $\mathrm{n}$ times the frequency change of $f_{\text {REF. }}$.

In Fig. 16, the coarse tunability within the $\mathrm{F}$ band (90-140 $\mathrm{GHz}$ ) is shown. It must be noted that the minimum selectable frequency is $f_{\text {REF }}$, while the maximum selectable frequency is limited by both the GS-OFCG optical span and the bandwidth of the photomixer.

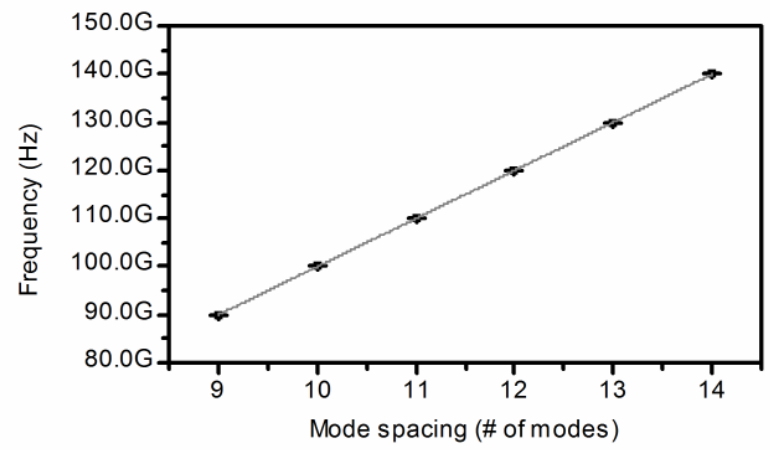

Fig. 16. Coarse tunability of $\mathrm{f}_{\mathrm{THz}}\left(\mathrm{f}_{\mathrm{REF}}=10 \mathrm{GHz}\right.$ ). $\mathrm{X}$ axis: $\mathrm{n}$ (number of optical modes spacing); $\mathrm{Y}$ axis: $\mathrm{THz}$ output frequency, $\mathrm{f}_{\mathrm{THz}}$. Measured data (average values: black dots; standard deviation: black caps); and linear fit (grey trace). Electrical spectra measurement parameters: $\operatorname{span}=2.1 \mathrm{kHz}$, $\mathrm{RBW}=30 \mathrm{~Hz}$, sweep time $=7 \mathrm{~s}$.

In Fig. 17, the fine tunability with $1.2 \mathrm{GHz}$ steps $\left(\right.$ of $\mathrm{f}_{\mathrm{THz}}$ ) is shown. This detuning has been chosen as it is the maximum range of tunability within the fine tunability range, that is, higher detuning would correspond to coarse tunability as the needed fine tunability range for complete continuous tunability is $\pm 5 \mathrm{GHz}$ of $\mathrm{f}_{\mathrm{THz}}$ detuning for a $\mathrm{f}_{\mathrm{REF}}$ of $10 \mathrm{GHz}$. This maximum range of fine tunability $\left(1.2 \mathrm{GHz} \mathrm{f}_{\mathrm{THZ}}\right.$ steps corresponds to a $\mathrm{f}_{\mathrm{REF}}$ detuning of $100 \mathrm{MHz}$ for $\mathrm{n}=12$ ) requires, as previously explained in section II, the PS to maintain the GS-OFCG characteristics and also the mode selection mechanism (see Fig. 10-13) has to be readjusted to re-lock the slave lasers.

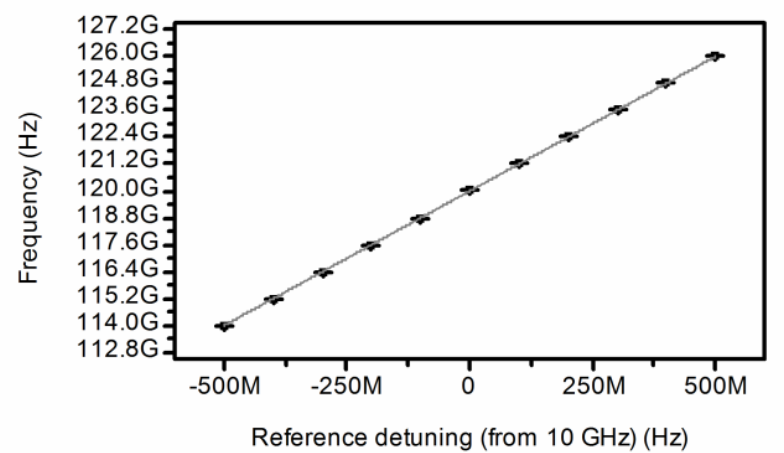

Fig. 17. Fine tunability, $1.2 \mathrm{GHz}$ steps of $\mathrm{f}_{\mathrm{THz}}\left(\mathrm{f}_{\mathrm{REF}}=10 \mathrm{GHz}\right) . \mathrm{X}$ axis: detuning of $\mathrm{f}_{\mathrm{REF}}$; $\mathrm{Y}$ axis: THz output frequency, $\mathrm{f}_{\mathrm{THz}}$. Measured data (average values: black dots; standard deviation: black caps); and linear fit (grey trace). Electrical spectra measurement parameters: $\operatorname{span}=2.1 \mathrm{kHz}, \mathrm{RBW}=30 \mathrm{~Hz}$, sweep time $=7 \mathrm{~s}$.

Fig. 18 shows a finer tunability characteristic of our system, in this case for very low frequency detuning values $(120 \mathrm{~Hz}$ steps of $\mathrm{f}_{\mathrm{THZ}}$ ). For this lower tuning range, there is neither need of PS adjustments nor OIL re-locking, as the frequency detuning is within the locking range. Due to the limited frequency accuracy readout of the ESA at this frequency range [48], smaller values of frequency detuning cannot be properly measured.

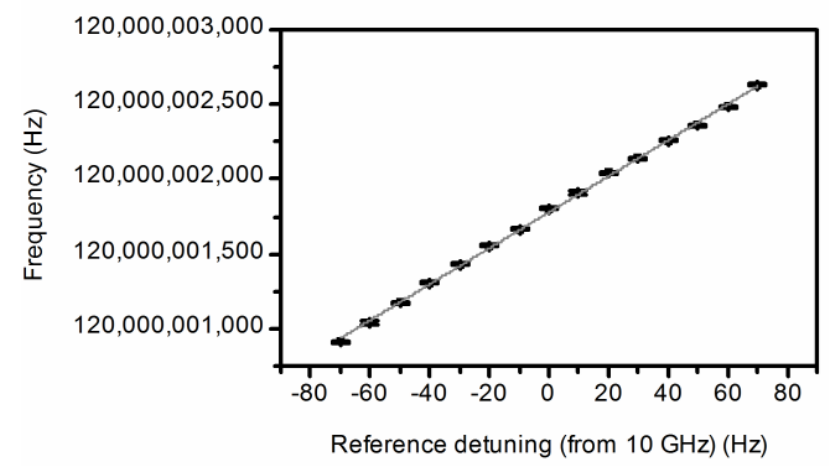

Fig. 18. Fine tunability, $120 \mathrm{~Hz}$ steps of $\mathrm{f}_{\mathrm{THZ}}\left(\mathrm{f}_{\mathrm{REF}}=10 \mathrm{GHz}\right) . \mathrm{X}$ axis: detuning of $\mathrm{f}_{\mathrm{REF}} ; \mathrm{Y}$ axis: THz output frequency, $\mathrm{f}_{\mathrm{THz}}$. Measured data (average values: black dots; standard deviation: black caps); and linear fit (grey trace). Electrical spectra measurement parameters: $\mathrm{span}=2.1 \mathrm{kHz}, \mathrm{RBW}=30 \mathrm{~Hz}$, sweep time $=7 \mathrm{~s}$.

The average values of the standard deviations of the set of samples of Fig. 16 to 20 are summarized in Table I. The coefficient of determination $\left(\mathrm{R}^{2}\right)$ of the linear fit of the data is also included. Almost a perfect linear behavior is achieved. Standard deviations in the range of the RBW $(30 \mathrm{~Hz}$ for the tunability test) and below the frequency readout accuracy are obtained. Thus, and due to the ESA limitations, we cannot conclude whether these fluctuations in the values are due to the system itself, or due to measurement instrumentation uncertainty [48].

TABLE I

ULTRA-HIGH RESOLUTION CONTINUOUS TUNABILITY

\begin{tabular}{lcl}
\hline \hline \multicolumn{1}{c}{ Range } & $\begin{array}{c}\text { Average standard } \\
\text { deviation }(\mathrm{Hz})\end{array}$ & $\mathrm{R}^{2}$ linear fit \\
\hline Coarse $\left(10 \mathrm{GHz}\right.$ steps of $\left.\mathrm{f}_{\mathrm{THz}}\right)$ & 22.27 & 1 \\
Fine $\left(1.2 \mathrm{GHz}\right.$ steps of $\left.\mathrm{f}_{\mathrm{THz}}\right)$ & 21.53 & 1
\end{tabular}


Simultaneous control of the coarse and fine tunability allows the synthesis of signals without dead regions and with full coverage of the whole frequency range under study (60-140 $\mathrm{GHz}$ ). Moreover, higher synthesized $\mathrm{THz}$ frequencies mean less PS adjustments to maintain the GS-OFCG characteristics as the detuning of $f_{\text {REF }}$ required for a same $\mathrm{THz}$ frequency change is smaller.

Finally, the tuning resolution of the synthesized signal is $\mathrm{f}_{\mathrm{THZ} \_ \text {RES }}=\mathrm{n} \cdot \mathrm{f}_{\text {REF_RES }}$, where $\mathrm{f}_{\text {REF_RES }}$ is the resolution of the reference $f_{\text {REF }}$. In our case, $f_{\text {REF_RES }}$ corresponds to $0.01 \mathrm{~Hz}$, which is translated to a tuning resolution of $0.12 \mathrm{~Hz}$ at 120 $\mathrm{GHz}$ (tuning resolution of $10^{-12}$ over the center frequency).

\section{High long-term frequency stability}

The frequency stability of the synthesized $\mathrm{THz}$ signal is measured as a function of the time. As expected from the stability analysis of the OIL mechanism (section IV), the synthesized $\mathrm{THz}$ signals feature an excellent long term stability. The electrical spectra variation of the sub- $\mathrm{THz}$ signal over one hour is measured. It has also been measured over the minimum span $(2.1 \mathrm{kHz}$ ), with a RBW of $30 \mathrm{~Hz}$ (in order to acquire more data in one hour). The variation of both peak frequency and peak power with respect to time is summarized in Table II. The power stability and especially the frequency stability are excellent. Their characterization has been again limited by the ESA, particularly for the power variation measurement, as the power accuracy in an ESA at high frequencies is low and its uncertainty is several dB's higher than the reported standard deviation $(1.5 \mathrm{~dB})$ [49].

TABLE II

LONG-TERM STABILITY (1 HOUR)

\begin{tabular}{lll}
\hline \hline \multirow{1}{*}{ Parameter } & \multicolumn{1}{c}{ Mean value } & Standard deviation \\
\hline Peak frequency & $120000001684.26 \mathrm{~Hz}$ & $5.2 \mathrm{~Hz}$ \\
Peak power & $-55.38 \mathrm{dBm}$ & $1.5 \mathrm{~dB}$ \\
\hline \hline
\end{tabular}

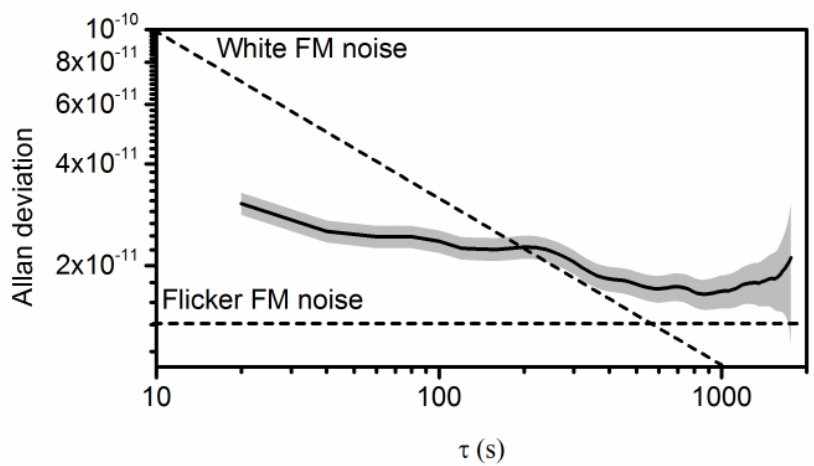

Fig. 19. Overlapped Allan deviation of the fractional frequency of the signal at $120 \mathrm{GHz}$. Black trace: overlapped Allan deviation; grey: error region.

Finally, the frequency stability is also studied through its Allan deviation [50]. Overlapped Allan deviation has been used and the results are shown in Fig. 19. The type of noise of the fluctuations is between white FM and Flicker FM [50]. Nevertheless, as the measurement instrumentation is not accurate enough for our system, we cannot confirm whether

the noise is due to the system itself or due to the measurement system (as is the case of the tunability analysis).

\section{CONCLUSION}

In this work, we have reported on a photonic system for generation of high quality $\mathrm{CW}$ sub-THz signals. The system is based on a Gain-Switching-based Optical Frequency Comb Generator (GS-OFCG), an optical mode selection mechanism and a n-i-pn-i-p superlattice photomixer. As mode selection mechanism, both selective tunable optical filtering using Fabry-Perot Tunable Filters (FPTF) and Optical Injection Locking (OIL) are evaluated. It is demonstrated that the OIL based method provides much better performance, especially with respect to long term frequency and power stability. The resulting system offers sub-THz signals with linewidth values $<10 \mathrm{~Hz}$ at $120 \mathrm{GHz}$, complete frequency range $(60-140 \mathrm{GHz})$ coverage with a resolution in the order of $0.1 \mathrm{~Hz}$ at $120 \mathrm{GHz}$ $\left(10^{-12}\right.$ of the generated frequency), and excellent long term frequency stability ( $5 \mathrm{~Hz}$ deviation over one hour). Most of these reported values are limited by the instrumentation accuracy and resolution, thus the actual values of the system could be below the data given in this paper. The frequency can be extended straightforwardly up to $1 \mathrm{THz}$. This scheme is compact, robust, and reliable. The excellent performance is particularly well suited for sub-THz photonic Local Oscillators and high resolution spectroscopy.

\section{REFERENCES}

[1] T. Nagatsuma, "Generating millimeter and terahertz waves," IEEE Microwave Magazine, vol. 10, no. 4, pp. 64-74, 2009.

[2] W. R. Tribe, D. A. Newnham, P. F. Taday, and M. C. Kemp, "Hidden object detection: security applications of terahertz technology," in SPIE 2004. Proceedings of SPIE, 2004, 1st ed., vol. 5354, pp. 168-176.

[3] E. Pickwell and V. P. Wallace, "Biomedical applications of terahertz technology," Journal of Physics D: Applied Physics, vol. 39, no. 17, pp. R301-R310, Sep. 2006.

[4] R. Piesiewicz, T. Kleine-Ostmann, N. Krumbholz, D. Mittleman, M. Koch, J. Schoebel, T. Kurner, and E. Felder, "Short-Range Ultra-Broadband Terahertz Communications: Concepts and Perspectives," IEEE Antennas and Propagation Magazine, vol. 49, no. 6, pp. 24-39, 2007.

[5] P. H. Siegel, "Terahertz technology," IEEE Transactions on Microwave Theory and Techniques, vol. 50, no. 3, pp. 910-928, 2002.

[6] A. G. Davies, A. D. Burnett, W. Fan, E. H. Linfield, and J. E. Cunningham, "Terahertz spectroscopy of explosives and drugs," Materials Today, vol. 11, no. 3, pp. 18-26, Mar. 2008.

[7] E. Bründermann, H.-W. Hübers, and M. F. Kimmitt, Terahert Techniques. Springer. Springer Series in Optical Sciences, Vol. 151, 2012, p. 383.

[8] R. E. Miles, X.-C. Zhang, H. Eisele, and A. Krotkus, "Terahertz Frequency Detection and Identification of Materials and Objects," in Terahertz Frequency Detection and Identification of Materials and Objects, 2007, p. 364.

[9] P. F. Taday, "Applications of terahertz spectroscopy to pharmaceutical sciences," Philosophical Transactions of the Royal Society of London. Series A: Mathematical, Physical and Engineering Sciences, vol. 362, no. 1815, pp. 351-364, Feb. 2004.

[10] P. F. Goldsmith and D. C. Lis, "Early Science Results From the Heterodyne Instrument for the Far Infrared (HIFI) on the Herschel Space Observatory," IEEE Transactions on Terahertz Science and Technology, vol. 2, no. 4, pp. 383-392, Jul. 2012. 
[11] I. Cámara Mayorga, A. Schmitz, T. Klein, C. Leinz, and R. Gusten, "First In-Field Application of a Full Photonic Local Oscillator to Terahertz Astronomy," IEEE Transactions on Terahertz Science and Technology, vol. 2, no. 4, pp. 393-399, Jul. 2012.

[12] Virginia Diodes Co., "Virginia Diodes Co. Multipliers," 2012. [Online]. Available: http://vadiodes.com/. [Accessed: 12-Feb2012].

[13] M. Feiginov, C. Sydlo, O. Cojocari, and P. Meissner, "Resonanttunnelling-diode oscillators operating at frequencies above 1.1 THz," Applied Physics Letters, vol. 99, no. 23, pp. 233503233506, 2011.

[14] R. Köhler, A. Tredicucci, F. Beltram, H. E. Beere, E. H. Linfield, A G. Davies, D. A. Ritchie, R. C. Iotti, and F. Rossi, "Terahertz semiconductor-heterostructure laser.," Nature, vol. 417, no. 6885, pp. 156-9, May 2002.

[15] D. Scherer, "The Art of Phase Noise Measurement," in $R F$ \& Microwave Measurement Symposium and Exhibition, 1983.

[16] M. Asada, "Theoretical analysis of spectral linewidth of terahertz oscillators using resonant tunneling diodes and their coupled arrays," Journal of Applied Physics, vol. 108, no. 3, p. 034504 , 2010.

[17] S. Suzuki, M. Asada, A. Teranishi, H. Sugiyama, and H. Yokoyama, "Fundamental oscillation of resonant tunneling diodes above $1 \mathrm{THz}$ at room temperature," Applied Physics Letters, vol. 97, no. 24, p. 242102, 2010.

[18] B. S. Williams, "Terahertz quantum-cascade lasers," Nature Photonics, vol. 1, no. 9, pp. 517-525, Sep. 2007.

[19] M. a. Belkin, F. Capasso, F. Xie, A. Belyanin, M. Fischer, A Wittmann, and J. J. Faist, "Room temperature terahertz quantum cascade laser source based on intracavity difference-frequency generation," Applied Physics Letters, vol. 92, no. 20, p. 201101, 2008.

[20] D. Rabanus, U. U. Graf, M. Philipp, O. Ricken, J. Stutzki, B. Vowinkel, M. C. Wiedner, C. Walther, M. Fischer, and J. Faist, "Phase locking of a 1.5 Terahertz quantum cascade laser and use as a local oscillator in a heterodyne HEB receiver.," Optics Express, vol. 17, no. 3, pp. 1159-68, Feb. 2009.

[21] Q. Qin, J. L. Reno, and Q. Hu, "MEMS-based tunable terahertz wire-laser over $330 \mathrm{GHz}$," Optics Letters, vol. 36, no. 5, p. 692, Feb. 2011.

[22] S. Preu, G. H. Döhler, S. Malzer, L. J. Wang, and A. C. Gossard, "Tunable, continuous-wave Terahertz photomixer sources and applications," Journal of Applied Physics, vol. 109, no. 6, pp. 061301-01 - 061301-56, 2011.

[23] E. Rouvalis, C. C. Renaud, D. G. Moodie, M. J. Robertson, and A. J. Seeds, "Continuous Wave Terahertz Generation From Ultra-Fast InP-Based Photodiodes," IEEE Transactions on Microwave Theory and Techniques, vol. 60, no. 3, pp. 509-517, Mar. 2012.

[24] Y. R. Huang, H. P. Chen, P. C. Chiu, J. I. Chyi, B. H. Wang, S. Y. Chen, and C. K. Sun, "Propagation, Resonance, and Radiation on Terahertz Optoelectronic Integrated Circuits," IEEE Photonics Journal, vol. 4, no. 3, pp. 1-1, 2012.

[25] Toptica Photonics, “Toptica Photonics. THz CW," 2012. [Online] Available: http://www.toptica.com. [Accessed: 12-Feb-2012].

[26] P. Acedo, H. Lamela, S. Garidel, C. Roda, J. P. Vilcot, G. Carpintero, I. H. White, K. A. Williams, M. Thompson, W. Li, M. Pessa, M. Dumitrescu, and S. Hansmann, "Spectral characterisation of monolithic modelocked lasers for mm-wave generation and signal processing," Electronics Letters, vol. 42, no. 16, pp. 928 929, 2006.

[27] A. R. Criado, P. Acedo, G. Carpintero, C. de Dios, and K. Yvind, "Observation of phase noise reduction in photonically synthesized sub-THz signals using a passively mode-locked laser diode and highly selective optical filtering," Optics Express, vol. 20, no. 2, pp. 1253-1260, Jan. 2012

[28] A. R. Criado, C. de Dios, P. Acedo, G. Carpintero, and K. Yvind, "Comparison of Monolithic Optical Frequency Comb Generators based on Passively Mode-Locked Lasers for Continuous Wave mmWave and sub-THz generation," Journal of Lightwave Technology, vol. 30, no. 19, pp. 3133-3141, Jan. 2012.

[29] R. Zhou, S. Latkowski, J. O'Carroll, R. Phelan, L. P. Barry, and P. M. Anandarajah, " $40 \mathrm{~nm}$ wavelength tunable gain-switched optical comb source," Optics Express, vol. 19, no. 26, pp. B415-20, Dec 2011.

[30] A. R. Criado, C. de Dios, G. H. Döhler, S. Preu, S. Malzer, S. Bauerschmidt, H. Lu, A. C. Gossard, and P. Acedo, "Ultra narrow linewidth CW sub-THz generation using GS based OFCG and n-ipn-i-p superlattice photomixers," Electronics Letters, vol. 48, no. 22, pp. 1425-1426, 2012.

[31] S. Ho-Jin, N. Shimizu, T. Furuta, K. Suizu, H. Ito, T. Nagatsuma, H. Song, and S. Member, "Broadband-Frequency-Tunable SubTerahertz Wave Generation Using an Optical Comb, AWGs, Optical Switches, and a Uni-Traveling Carrier Photodiode for Spectroscopic Applications," Journal of Lightwave Technology, vol. 26, no. 15, pp. 2521-2530, 2008.

[32] C. de Dios, A. R. Criado, G. H. Döhler, S. Preu, S. Malzer, S. Bauerschmidt, L. E. García, P. Acedo, and D. Segovia, "Sub-THz and $\mathrm{THz}$ Photonic Generation with Continuous Tunability Using Gain Switching based Optical Frequency Comb Generators and n-ip-n-i-p Superlattice Photomixers," in International Topical Meeting on Microwave Photonics. MWP 2012, 2012.

[33] E. Rouvalis, C. C. Renaud, D. G. Moodie, M. J. Robertson, and A. J. Seeds, "Traveling-wave Uni-Traveling Carrier photodiodes for continuous wave THz generation," Optics express, vol. 18, no. 11, pp. 11105-10, May 2010.

[34] S. Preu, F. H. Renner, S. Malzer, G. H. Döhler, L. J. Wang, M. Hanson, A. C. Gossard, T. L. J. Wilkinson, E. R. Brown, and G. H. Döhler, "Efficient terahertz emission from ballistic transport enhanced n-i-p-n-i-p superlattice photomixers," Applied Physics Letters, vol. 90, no. 21, p. 212115, 2007.

[35] S. Bauerschmidt, S. Preu, S. Malzer, G. H. Döhler, L. J. Wang, H. $\mathrm{Lu}$, and A. C. Gossard, "Continuous wave Terahertz emitter arrays for spectroscopy and imaging applications," in SPIE Proceedings Vol. 7671 Terahertz Physics, Devices, and Systems IV: Advanced Applications in Industry and Defense, 2010, p. 76710D-11.

[36] P. P. Vasil'ev, I. H. White, and J. Gowar, "Fast phenomena in semiconductor lasers," Reports on Progress in Physics, vol. 63, p. 1997, 2000.

[37] R. Wu, V. R. Supradeepa, C. M. Long, D. E. Leaird, and A. M. Weiner, "Generation of very flat optical frequency combs from continuous-wave lasers using cascaded intensity and phase modulators driven by tailored radio frequency waveforms," Optics Letters, vol. 35, no. 19, pp. 3234-3236, Sep. 2010.

[38] M. Song, R. Wu, V. Torres-Company, and A. M. Weiner, "Programmable Microwave Photonic Phase Filters With Large Time-Bandwidth Product Based on Ultra- Broadband Optical Frequency Comb Generation," in IEEE International Topical Meeting on Microwave Photonics. MWP 2012, 2012, 2012.

[39] C. He, S. Pan, R. Guo, Y. Zhao, and M. Pan, "Ultraflat optical frequency comb generated based on cascaded polarization modulators," Optics Letters, vol. 37, no. 18, pp. 3834-3836, Sep. 2012.

[40] V. R. Supradeepa and A. M. Weiner, "Bandwidth scaling and spectral flatness enhancement of optical frequency combs from phase-modulated continuous-wave lasers using cascaded four-wave mixing," Optics Letters, vol. 37, no. 15, pp. 3066-3068, Aug. 2012.

[41] C. de Dios and H. Lamela, "Improvements to Long-Duration LowPower Gain-Switching Diode Laser Pulses Using a Highly Nonlinear Optical Loop Mirror: Theory and Experiment," Journal of Lightwave Technology, vol. 29, no. 5, pp. 700-707, Mar. 2011.

[42] M. Egan, "The 20-Bit DAC Is the Easiest Part of a 1-ppm-Accurate Precision Voltage Source," Analog Dialogue, vol. 44, no. 04, 2010.

[43] L. L. Wang and T. Kowalcyzk, "A Novel Locking Technique for Very Narrow Tunable Optical Filters With Sub-GHz 3-dB Bandpass," IEEE Photonics Technology Letters, vol. 22, no. 17, pp. $1267-1269$, Sep. 2010

[44] M. P. van Exter, S. J. M. Kuppens, and J. P. Woerdman, "Excess phase noise in self-heterodyne detection," IEEE Journal of Quantum Electronics, vol. 28, no. 3, pp. 580 - 584, 1992.

[45] ILX Lightwave Co., "Tech Note: Typical Output Stability of the LDC-3724B," 2012

[46] A. Hirata, H. Togo, N. Shimizu, H. Takahashi, K. Okamoto, and T. Nagatsuma, "Low-phase noise photonic millimeter-wave generator 
using an AWG integrated with a 3-dB combiner," Transactions on Electronics, IEICE, vol. E88C, no. 7, pp. 1458-1464, 2005.

P. Gallion and G. Debarge, "Quantum phase noise and field correlation in single frequency semiconductor laser systems," IEEE Journal of Quantum Electronics, vol. 20, no. 4, pp. 343-349, Apr. 1984.

[48] Anritsu Co., "MS2668C Spectrum Analyzer Operation Manual Vol. $1, " 2007$.

[49] Agilent Technologies, "Product Note. Agilent PSA HighPerformance Spectrum Analyzer Series. Amplitude Accuracy." 2006.

[50] W. J. Riley, NIST Special Publication 1065: Handbook of Frequency Stability Analysis. National Institute of Standards and Technology (NIST), 2008.

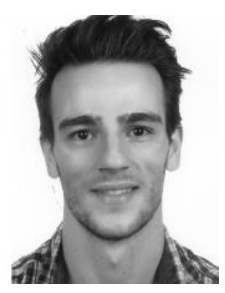

Á. R. Criado (S’09) received his $\mathrm{BSc}$ and $\mathrm{MSc}$ on Telecommunication Engineering from Universidad Carlos III de Madrid (UC3M, Spain) in 2009, his MRes on Advanced Electronics Systems from UC3M in 2011 and his PhD from UC3M in 2013.

$\mathrm{He}$ has been involved in research tasks on laser diagnostics for fusion plasmas and he worked as collaborator researcher during 2009 and 2010 in the National Fusion Laboratory in CIEMAT (Spain).

Their main research interests lie in the field of low noise photonic generation, homodyne/heterodyne detection of mm-waves and sub- $\mathrm{THz}$ signals and Optical Frequency Comb generation.

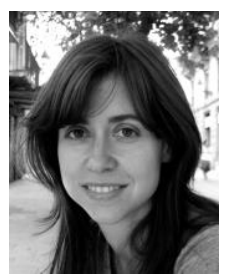

C. de Dios received her M.S. degree in Applied Physics and Electronics from the Universidad Complutense of Madrid. Then, she joined the private sector as a Technical Consultant for Hewlett-Packard. Since 2002, she has been a member of the Optoelectronics and Laser Technology Group at the Universidad Carlos III de Madrid, where she obtained her MRes in 2004 and her Doctorate in 2010 for her work in ultrafast pulsed diode lasers and nonlinear pulse compression. Now, she is an assistant professor at the Electronics Technology Department, in this same university. Her research interests are high-speed optical communications, pulsed semiconductor laser sources, nonlinear optical phenomena and subterahertz and millimeter wave photonic signal synthesis and detection.

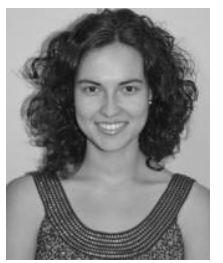

E. Prior received her $\mathrm{BSc}$ and $\mathrm{MSc}$ on Telecommunication Engineering from Universidad Carlos III de Madrid (Spain) in 2012. From 2011, she has been involved in research and development tasks related to Microwave Photonics and Radio over Fiber, in both transmission and receiver systems. She currently works on pulsed operation (Gain Switching) on VCSELs at Universidad Carlos III de Madrid.

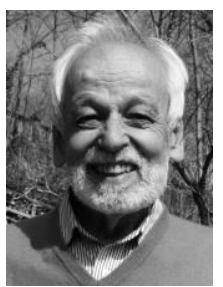

G. H. Döhler received his Ph.D. in physics from the Technical University in Munich (Germany) in 1968. He has worked with the Max-Planck-Institute in Stuttgart and with HP-Labs in Palo Alto, before taking a position as Professor at the University of Erlangen-Nürnberg (Germany). After retiring he joined the Max Planck Institute for the Science of Light in 2009. Most of his research has been devoted to experimental and theoretical investigations of transport and optical properties of superlattices, nanostructures, nanodevices and quantum dots Most recently he has worked on femtosecond transport in nanostructures and its application to $\mathrm{THz}$ emitters. He has (co)-authored more than 400 publications including many reviews, book chapters, and patents. G. H. Döhler is the recipient of the Walter-Schottky-Prize of the German Physical Society 1984. In 2004 a Sarojini Damodaran Fellowship by the Tata Institute of Fundamental Research (Mumbai) was awarded to him and in 2011 a Chair of Excellence by the University Carlos III in Madrid.

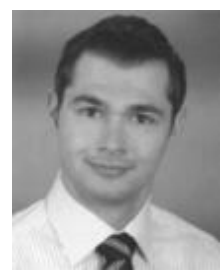

S. Preu received the Diploma degree and the Ph.D. degree in physics (summa cum laude) from the Univ. of Erlangen-Nuremberg, Erlangen, Germany, in 2005 and 2009, respectively. From 2004 to 2010, he was with the Max Planck Institute for the Science of Light, Erlangen, Germany. During 2010-2011, he worked at the Materials and Physics Department, University of Santa Barbara, CA. Currently, he is at the Chair of Applied Physics, University Erlangen-Nuremberg, Erlangen, Germany. His research interests focus on the development of semiconductor-based $\mathrm{THz}$ sources and detectors. $\mathrm{He}$ also works on applications of $\mathrm{THz}$ radiation, in particular spectroscopy, and the characterization of novel $\mathrm{THz}$ optics and materials such as whispering gallery mode resonators and graphene.

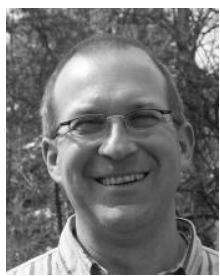

S. Malzer received his $\mathrm{PhD}$ in semiconductor physics in 1991 in Erlangen, Germany. He has been a staff member at the Institute of condensed matter physics at the University of Erlangen. There, he has been involved in III-V- optoelectronic research projects on quantum dots, high speed opto-electronics, and $\mathrm{THz}$ devices. Currently, his research focus is in the $\mathrm{THz}$-generation by photomixing in semiconductor nanostructures and applications of $\mathrm{CW} \mathrm{THz}$ tunable devices. $\mathrm{He}$ is coauthor of more than 100 papers in international journals and conference proceedings and holds one patent.

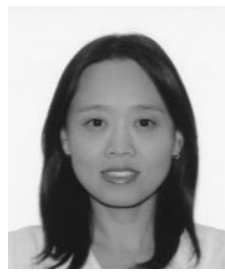

H. Lu received her B. S. degree in Chemistry from University of Science and Technology of China, and Ph.D in Chemistry from the City University of New York in 2007. Her Ph. D research was focused on intersubband transitions of wide bandgap II-VI semiconductors grown by MBE. She is currently working in University of California at Santa Barbara (UCSB) as a project scientist. Her current research areas include using and developing $\mathrm{MBE}$ growth techniques for synthesis of materials and material structures, and characterization and processing for fundamental understanding and device applications, especially applications in thermoelectrics. Other research interests include two dimensional electron gas for electronic spin states control and rare earth-V compounds in III-V semiconductors for ultrafast photoconductive switches and $\mathrm{THz}$ based technology. Dr. Lu has coauthored in more than 40 papers in refereed journals.

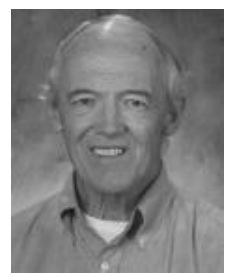

A. C. Gossard (SM'88-F'01-LF'12) is Professor of Materials and Electrical and Computer Engineering at University of California, Santa Barbara. His special interests are molecular beam epitaxy, the growth of quantum wells and superlattices, and their applications to high performance electrical and optical devices. Dr. Gossard is a fellow of the American Physical Society, AAAS, and a member of the National Academy of Engineering, and the National Academy

of Sciences

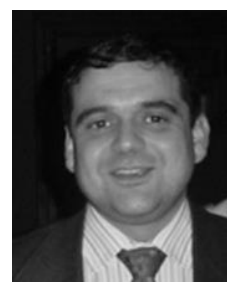

P. Acedo (M'99) received his bachelor degree on Telecommunication Engineering in 1993 from the Universidad Politécnica de Madrid, and his Doctorate (with honors) from the Universidad Carlos III de Madrid in 2000 for his work on heterodyne two color laser interferometry for fusion plasma diagnostics at the Stellarator TJ-II, (Laboratorio Nacional de Fusión, CIEMAT, Madrid) and Tokamak C-Mod (Plasma Science and Fusion Centre, Massachusetts Institute of Technology). In 2002 he was appointed as Assistant Professor by Universidad Carlos III de Madrid where he has continued with the development of scientific instrumentation systems for fusion plasma diagnostics and biomedical applications. He has been also involved in research on high speed semiconductor laser devices for microwave and millimeter-wave generation and processing. Currently his interests in this line are the development of integrated active photonic antennas for mm-wave and $\mathrm{THz}$ generation and detection. 